\title{
Adjuvant therapy for renal cell carcinoma
}

\author{
Paramvir Sawhney ${ }^{1}$, Suyanto Suyanto ${ }^{2}$, Agnieszka Michael $^{2}$, Hardev Pandha ${ }^{2}$ \\ 'Department of Oncology, University College London Cancer Institute, London WC1E 6DD, UK. \\ ${ }^{2}$ St Luke's Cancer Centre, Royal Surrey County Hospital, Guildford GU2 7XX, UK. \\ Correspondence to: Dr. Hardev Pandha, St Luke's Cancer Centre, Royal Surrey County Hospital, Egerton Road, Guildford GU2 \\ 7XX, UK. E-mail: h.pandha@surrey.ac.uk
}

How to cite this article: Sawhney P, Suyanto S, Michael A, Pandha H. Adjuvant therapy for renal cell carcinoma. J Cancer

Metastasis Treat 2021;7:48. https://dx.doi.org/10.20517/2394-4722.2021.64

Received: 15 Mar 2021 First Decision: 25 May 2021 Revised: 16 Jun 2021 Accepted: 29 Jun 2021 First online: 4 Jul 2021

Academic Editors: Lucio Miele, Hendrik Van Poppel Copy Editor: Yue-Yue Zhang Production Editor: Yue-Yue Zhang

\begin{abstract}
Recent advances in the treatment of metastatic renal cell carcinoma expose a gap in the treatment of less advanced, localized disease. Tyrosine kinase inhibitors, which revolutionized the treatment of metastatic disease, have not provided a similar survival benefit in the adjuvant setting and currently only sunitinib is approved by the Food and Drug Administration for adjuvant treatment in patients with high-risk of recurrence based on S-TRAC disease-free survival data. The advent of immune checkpoint inhibitors has offered a fresh hope in the field of adjuvant treatment after encouraging results are seen with combination of immune checkpoint inhibitors as well as with targeted therapy in the metastatic setting. Several studies are investigating these combinations in the adjuvant setting, and it is hoped that they will bring about a better outcome for a largely unmet need in kidney cancer treatment.
\end{abstract}

Keywords: Localized renal cell carcinoma, adjuvant, immunotherapy, tyrosine kinase inhibitors, prognosis, clinical trials, predictive model, risk stratification

\section{INTRODUCTION}

Localized renal cell carcinoma (RCC) even if treated radically, with surgery, has a high recurrence rate of $30 \%$ to metastatic disease, which carries a poor prognosis. There is an unmet need for adjuvant systemic therapies which can effectively delay recurrence, or increase the cure rate, and, unsurprisingly, this is a highly active area of clinical trial work. Unfortunately, the silver-bullet has not yet arrived, with vascular

The Author(s) 2021. Open Access This article is licensed under a Creative Commons Attribution 4.0 International License (https://creativecommons.org/licenses/by/4.0/), which permits unrestricted use, sharing, adaptation, distribution and reproduction in any medium or format, for any purpose, even commercially, as long as you give appropriate credit to the original author(s) and the source, provide a link to the Creative Commons license, and indicate if changes were made. 
endothelial growth factor receptor (VEGFR) tyrosine kinase inhibitors (TKIs) convincingly failing to provide benefit, across no fewer than six large phase 2-3 trials. One important caveat is sunitinib, which has been approved by the US Food and Drug Administration (FDA) in the adjuvant setting post-nephrectomy in those with high risk of recurrence based on the Sunitinib as Adjuvant Treatment for High-risk Renal Cell Carcinoma Following Nephrectomy (S-TRAC) study, a trial which is discussed below. Thus, the results of several phase 3 trials involving immune-checkpoint inhibitors (ICIs) are eagerly awaited. It is likely that differential tumor biology between metastatic disease and localized disease has a significant role to play in the failure of TKIs. There are several validated clinical models which are prognostic for recurrence to metastatic disease after surgery or overall survival. These stratify patients into risk groups which are used for patient selection into clinical trials, and they are discussed in the next section. In all the trials, we discuss below, the disease-free survival (DFS) has been chosen as a primary endpoint due to long durations of follow-up required to accumulate events with overall survival (OS) as an endpoint, although it is contentious as to whether DFS represents a good surrogate marker for OS in the adjuvant setting.

\section{RISK STRATIFICATION AND PROGNOSTIC MODELS}

There are several validated risk stratification and prognostic models for use in localized RCC, designed for the postoperative setting. The SSIGN (Stage, Size, Grade, and Necrosis) score was developed in 2002 using a cohort of 1801 patients with localized clear-cell RCC (ccRCC) treated with radical nephrectomy from 1970 to 1988. It found TNM stage, size, nuclear grade, and tumor necrosis to significantly correlate with cancer specific survival ${ }^{[1]}$. This model retained its prognostic ability when evaluated against more recent patients (1999-2010) undergoing both radical and partial nephrectomy ${ }^{[2]}$.

The UCLA Integrated Staging System (UISS) was developed in 2001 and validated on a smaller set of patients, 661, who had nephrectomies between 1989 and 1999 where the TNM stage, Fuhrman's grade, and Eastern Cooperative Oncology Group (ECOG) performance status was found to be prognostic for survival. The model stratifies patients into 5 survival groups designated UISS I- $\mathrm{V}^{[3]}$. Around the time the UISS model was developed, the Memorial Sloan Kettering Cancer Center postoperative nomogram was also developed and validated against 601 RCC patients treated with nephrectomy, to predict 5-year recurrence after surgery. Symptoms, histology, tumor size, and pathological stage are combined to create a score out of 180 which is prognostic for recurrence at five years ${ }^{[4]}$.

Later, the Leibovich score, developed in 2003, was validated against 1671 patients with ccRCC undergoing nephrectomy between 1970 and 2000 to predict progression to metastatic RCC (mRCC). Tumor stage, tumor size, regional lymph node status, nuclear grade, and histologic tumor necrosis were all determined as being prognostic, and a scoring algorithm was developed based on these factors ${ }^{[5]}$.

\section{ADJUVANT TREATMENTS - PAST, PRESENT, AND FUTURE Interferon-alpha}

In the 1990s and 2000s, interferon-alpha was evaluated in the adjuvant setting after promising results in the mRCC setting in the 1980s. However, two trials failed to show benefit. In 2001, Pizzocaro et al. ${ }^{[6]}$ published results of a randomized trial of 247 patients with Robson stage II and III RCC comparing adjuvant recombinant interferon-alpha $2 \mathrm{~b}$ with observation post-radical nephrectomy. The trial showed the resulting 5-year OS probability was 0.665 for treated patients vs. 0.660 for controls, while the event-free survival (EFS) probability was 0.671 for treated patients $v s$. 0.567 for controls. These differences were not statistically significant, with a $2 \mathrm{P}$ value of 0.861 for $\mathrm{OS}$ and a $2 \mathrm{P}$ value of 0.107 for EFS. Interestingly, interferon-alpha was found to have a harmful effect in 97 pNo patients but a beneficial effect in a small subset of 13 cases of $\mathrm{pN} 2 / \mathrm{pN} 3$ patients compared with 13 controls. This finding might be useful as a hypothesis that there was an 
interaction between pathologic node status and treatment response, but the finding could not be relied upon to change clinical practice due to the small sample size ${ }^{[6]}$.

In 2003, Messing et al. ${ }^{[7]}$ published the results of a phase 3 trial evaluating interferon-alpha in the setting of resectable RCC. The study involved 283 patients with $\mathrm{pT} 3-4 \mathrm{a}$ and/or node positive disease randomly assigned to interferon-alpha-NL or observation after radical nephrectomy and lymphadenectomy. At a median follow-up of 10.4 years, the median OS was 5.1 years in the treated group vs. 7.4 years in the observation group $(P=0.9)$ and median relapse-free survival (RFS) was 2.2 years in the cohort receiving interferon-alpha vs. 3.0 years in the group only under observation $(P=0.33)^{[7]}$.

\section{Interleukin-2}

After the aforementioned trials, low-dose interleukin-2 plus interferon-alpha was evaluated by Passalacqua et al. ${ }^{[8]}$ in a randomized, phase 3 trial in which 310 patients were randomized to receive interleukin-2 plus interferon-alpha or observation after primary surgery for RCC, with 303 patients evaluable in the intention-to-treat analyses. At a median follow-up of 54 months, the hazard ratios (HRs) were 1.07 for OS [ $95 \%$ confidence interval $(\mathrm{CI})$ : 0.64-1.79, $P=0.79$ ] and 0.84 for RFS (95\%CI: 0.54-1.31, $P=$ 0.44). However, despite this overall lack of benefit, an unplanned sub-group analysis found that the treatment arm patients with $\geq 2$ of the factors age $\leq 60$ years, pNo, tumor grade $1-2$, and pT 3 a stage had an improved RFS with a HR of 0.44 (95\%CI: 0.24-0.82, $P \leq 0.01$ ), while patients with $<2$ of these factors had a significantly poorer $\mathrm{OS}(\mathrm{HR}=2.27,95 \% \mathrm{CI}: 1.03-5.03, P=0.037)$, suggesting that patient stratification could uncover patients most likely to benefit from adjuvant treatment ${ }^{[8]}$.

\section{Girentuximab}

Girentuximab is a monoclonal antibody which binds to carbonic anhydrase-IX (CA-IX), a transmembrane glycoprotein expressed on the surface of most RCC cells, and it triggers antibody-dependent cell-mediated cytotoxicity, in a process thought to be mediated by natural killer cells and other immune effector cells. The phase 3 randomized ARISER trial evaluated girentuximab in the setting of ccRCC with high risk of recurrence after nephrectomy. In total, 864 patients were randomized to receive girentuximab or placebo, but the study unfortunately found no statistically significant difference in DFS (HR $=0.97,95 \%$ CI: 0.791.18) or OS (HR $=0.99,95 \% \mathrm{CI}: 0.74-1.32)$ between the cohorts ${ }^{[9]}$.

\section{Tyrosine kinase inhibitors}

The arrival of TKIs targeting VEGFRs in the 2000s changed the treatment paradigm for mRCC. Since then, there has been interest in whether this benefit would translate to the adjuvant setting, based on the knowledge that metastatic disease is highly vascularized and that biallelic inactivation of the VHL gene, a tumor suppressor gene, appears to be an initiating event in sporadic ccRCCs which subsequently drives $V E G F A$ gene over-expression and thus angiogenesis ${ }^{[10]}$. Several cohort studies have shown that VHL gene inactivation is present in the majority of sporadic ccRCCs, which supports the rationale of targeting VEGFA and VEGFR in RCC. With the relative success of VEGFR TKI therapy in the metastatic setting, it was hoped that this strategy could be replicated in the adjuvant setting ${ }^{[1]}$.

Unfortunately, after five large randomized controlled trials, VEGFR TKIs have not been shown to improve survival outcomes in the adjuvant setting. Indeed, a recent meta-analysis of four of these trials showed no improvement in DFS for patients receiving VEGFR TKIs after curative intent nephrectomy, although a slight improvement in DFS was observed in higher-risk patients, opening the possibility that improved patient stratification may uncover those who are likely to benefit more from adjuvant treatment ${ }^{[12]}$. Table 1 shows the key inclusion criteria of different adjuvant VEGFR TKI trials. 
Table 1. Key features of adjuvant VEGFR TKI trials

\begin{tabular}{|c|c|c|c|c|c|}
\hline & ASSURE $(n=1943)$ & S-TRAC $(n=615)$ & PROTECT $(n=1538)$ & ATLAS $(n=724)$ & SORCE $(n=1711)$ \\
\hline Comparator Arms & Sunitinib: Sorafenib: Placebo & Sunitinib: Placebo & Pazopanib: Placebo & Axitinib: Placebo & Sorafenib: Placebo \\
\hline Histology & ccRCC or non-ccRCC & preponderant ccRCC & ccRCC or predominant ccRCC & $\begin{array}{l}\text { Preponderant, defined as > } \\
50 \%, \text { ccRCC }\end{array}$ & ccRCC or non-ccRCC \\
\hline Key inclusion criteria & $\begin{array}{l}\text { AJCC 6th ed.: } \\
\text { - pT1b, G3-4, NO (or pNX, clinical NO); } \\
\text { - pT2, G any, NO (or pNX, clinical NO); } \\
\text { - pT3, G any, NO (or pNX, clinical NO); } \\
\text { - pT4, G any, NO (or pNX, clinical NO); } \\
\text { - T any, G any, N+ (fully resected) } \\
\text { RV microvascular invasion any grade/stage (provided } \\
\text { MO) } \\
\text { Removal of all positive LNs; } \\
\text { Negative margins, but positive RV margins eligible unless } \\
\text { invasion of RV wall at the margin (provided no other } \\
\text { margins }+ \text { ) }\end{array}$ & $\begin{array}{l}\text { High risk per modified } \\
\text { UISS criteria: } \\
\text { - } \mathrm{pT2} \text {, NO (grades 3-4); } \\
\text { - } \mathrm{pT3}-4, \text { NO; } \\
\text { - } \mathrm{pTx} \text { N1 } \\
\text { No macroscopic residual } \\
\text { or metastatic disease } \\
\text { Microscopic disease (R1) } \\
\text { are acceptable }\end{array}$ & $\begin{array}{l}\text { AJCC TNM staging v. 2010, } \\
\text { and Fuhrman nuclear grading: } \\
\text { - pT2, G3 or G4, NO; } \\
\text { - pT3, G any, NO; } \\
\text { - pT4, G any, NO; } \\
\text { - pT any, G any, N1 }\end{array}$ & $\begin{array}{l}\text { AJCC TNM staging v. } \\
\text { 2010: } \\
\text { - pT2, pNO or pNx; } \\
\text { - pT3, pNO or pNx; } \\
\text { - pT4, pNO or pNx; } \\
\text { - Any pT, pN1 } \\
\text { No macroscopic residual } \\
\text { or metastatic disease }\end{array}$ & $\begin{array}{l}\text { Intermediate- or high-risk disease, } \\
\text { i.e., Leibovich score } 3 \text { to } 11\end{array}$ \\
\hline Treatment duration & 54 weeks & 1 year & 1 year & $1-3$ years & 1 year or 3 years \\
\hline $\begin{array}{l}\text { Treatment discontinuation } \\
\text { due to TRAEs (\%) }\end{array}$ & 44 (sunitinib) vs. 45 (sorafenib) vs. 11 (placebo) & $\begin{array}{l}28.1 \text { (sunitinib) vs. } 5.6 \\
\text { (placebo) }\end{array}$ & $\begin{array}{l}35 \text { (pazopanib } 600 \mathrm{mg} \text { ) vs. } 5 \\
\text { (placebo } 600 \mathrm{mg}) ; \\
39 \text { (pazopanib } 800 \mathrm{mg} \text { ) vs. } 6 \\
\text { (placebo } 800 \mathrm{mg})\end{array}$ & $\begin{array}{l}23 \text { (axitinib) vs. } 11 \\
\text { (placebo) }\end{array}$ & $\begin{array}{l}30 \text { (1-year sorafenib) vs. } 34 \text { (3-year } \\
\text { sorafenib) vs. } 4 \text { (placebo) }\end{array}$ \\
\hline Grade 3-4 TRAEs (\%) & 63 (sunitinib) vs. $72 \%$ (sorafenib) vs. $25 \%$ (placebo) & $\begin{array}{l}60.5 \text { (sunitinib) vs. } 19.4 \\
\text { (placebo) }\end{array}$ & $\begin{array}{l}66 \text { (pazopanib) vs. } 21 \\
\text { (placebo) }\end{array}$ & $\begin{array}{l}61 \text { (axitinib) vs. } 30 \\
\text { (placebo) }\end{array}$ & $\begin{array}{l}58.6 \text { (1-year sorafenib) vs. } 63.9 \text { (3- } \\
\text { year sorafenib) vs. } 29.2 \text { (placebo) }\end{array}$ \\
\hline
\end{tabular}

TRAEs: Treatment-related adverse events; LNs: lymph nodes; RV: renal vein.

A significant problem encountered in the recent VEGFR TKI trials is the toxicity of treatment, which has led to both dose reductions and discontinuation of treatment (often despite dose reductions). This brought to the fore that in the adjuvant setting what a patient will tolerate as an adverse effect on their quality of life resulting from treatment is often less than what a patient will accept in the metastatic setting where the perceived benefit of treatment is much greater. Moreover, the harm to benefit ratio in the adjuvant setting might favor stopping treatment or dose reducing on clinical grounds at a lower toxicity threshold than in the metastatic setting. It is also worth noting that, when sunitinib, pazopanib, and axitinib were evaluated in metastatic trials, the overall and Grade 3 or above adverse event (AE) rates were much lower than when the same drugs were used in adjuvant trials, which partly explains the significantly lower treatment discontinuation rates due to AEs in these metastatic trials ${ }^{[13-16]}$. 
The ASSURE trial, which was the first VEGFR inhibitor trial to report, evaluated sunitinib and sorafenib in the setting of high-grade, T1b or greater, completely resected non-mRCC. The study randomized 1943 patients to sunitinib, sorafenib, or placebo. There was no survival benefit seen with either agent, but a significant treatment discontinuation rate occurred despite dose reductions as a result of toxicity ${ }^{[17]}$. Median DFS was 5.8 years for sunitinib with a HR of 1.02 (97.5\%CI: $0.85-1.23, P=0.8038)$ and 6.6 years for placebo. Sorafenib also failed to show benefit with a median DFS of 6.1 years $(\mathrm{HR}=0.97,97.5 \% \mathrm{CI}$ : 0.80-1.17, $P=$ 0.7184 ). The treatment discontinuation rate due to AEs was $44 \%$ for patients who received sunitinib, and this was a common theme across all VEGFR TKI adjuvant trials, which had higher rates of treatment discontinuation due to treatment-related AEs, in terms of both frequency and severity, than when the same drugs were investigated in the metastatic setting ${ }^{[13-16]}$.

The S-TRAC randomized phase 3 trial evaluated sunitinib in the setting of locoregional, high-risk ccRCC after nephrectomy. It enrolled 615 patients who were randomized to receive either sunitinib or placebo. Median DFS was 6.8 years (95\%CI: 5.8 to not reached) in the sunitinib cohort vs. 5.6 years (95\%CI: 3.8-6.6) in the placebo cohort ( $\mathrm{HR}=0.76,95 \% \mathrm{CI}$ : 0.59-0.98, $P=0.03)$, demonstrating DFS benefit. However, it is notable that the DFS benefit came at the expense of toxicity with $34.5 \%$ of patients in the sunitinib group requiring dose reductions due to AEs vs. $2 \%$ of patients receiving placebo. Similarly, dose reductions occurred more frequently in the sunitinib group at $34.3 \% v s .2 \%$, as did treatment stoppage at $28.1 \% v s$. $5.6 \%$, respectively. The sunitinib group also had a higher frequency of Grade 3 or 4 AEs at $48.4 \%$ for Grade 3 and $12.1 \%$ for Grade 4 vs. $15.8 \%$ and $3.6 \%$ in the placebo cohort, respectively ${ }^{[18]}$. Although the trial failed to demonstrate an OS benefit, based on the DFS data, sunitinib was FDA approved in November 2017 for adjuvant treatment in high-risk adult patients.

The PROTECT randomized phase 3 trial evaluated pazopanib in the setting of high-risk, locally advanced RCC after nephrectomy. It enrolled 1538 patients who were randomized to receive pazopanib or placebo for one year. The initial starting dose of $800 \mathrm{mg}$ pazopanib had to be reduced to $600 \mathrm{mg}$ because of a high treatment discontinuation rate, echoing the problems encountered in the ASSURE trial. Once again, in a TKI trial, DFS analysis showed no benefit of pazopanib over placebo. Treatment discontinuations as a result of AEs were $35 \%$ and $39 \%$ in the pazopanib 600 and $800 \mathrm{mg}$ groups in comparison with $5 \%$ and $6 \%$ for the placebo 600 and $800 \mathrm{mg}$ groups, respectively ${ }^{[19]}$.

The ATLAS randomized phase 3 trial evaluated axitinib in the setting of locoregional RCC at risk of recurrence after nephrectomy. The study enrolled 724 patients who were randomized to receive axitinib or placebo. The trial did not meet its primary endpoint of DFS, and there were more Grade 3 or 4 AEs in the axitinib group vs. placebo, $61 \%$ vs. $30 \%$, respectively. With axitinib vs. placebo, the AEs led to dose reductions in $56 \% v s .8 \%$ of patients, dose interruptions in $51 \% v s .22 \%$, and permanent treatment discontinuation in $23 \% v s .11 \%$, respectively. However, in the highest-risk sub-group, a $36 \%$ reduction in risk of DFS events was observed per investigator (HR $=0.641,95 \% \mathrm{CI}$ : 0.468-0.879, $P=0.0051$ ), once again highlighting the importance of patient stratification ${ }^{[20]}$.

The SORCE phase 3 trial enrolled 1711 post-nephrectomy RCC patients with intermediate to high risk of recurrence who were randomly assigned to receive three years of placebo, one year of sorafenib followed by two years of placebo, or three years of sorafenib. No differences in DFS in all groups or subgroups were found. Moreover, despite treatment adaptations, over half the patients stopped their treatment by 12 months, with $24 \%$ of patients who received sorafenib reporting Grade 3 hand-foot skin reactions. In addition, the restricted mean survival time was 6.81 years for three years of sorafenib vs. 6.82 years for placebo (95\%CI: 0.49-0.48 years, $P=0.99)^{[21]}$. As the latest of all the VEGFR TKI trials to report, SORCE 
presented a final signal that perhaps a different approach to tackling the problem of recurrence after surgery is needed.

\section{Immune checkpoint inhibitors}

ICIs either alone or in combination with TKIs have been shown to be efficacious in the mRCC setting with tolerable side-effect profiles. There is great interest, with studies ongoing, in whether this benefit will translate to the adjuvant setting, especially given the prior failure of TKIs to demonstrate benefit. Indeed, the biological rationale for enhancing the immune tumor response through programmed cell death protein 1 (PD-1)/programmed death ligand 1 (PDL-1) blockade may represent a more efficacious approach than antiangiogenic blockade, particularly in clearing circulating tumor cells or micrometastases. Anti-PD-1/PDL1 ICIs work by disrupting the expressed tumor surface PD-L1 neoantigen inhibitory response when it binds to PD-1 T-cells that leads to immune tolerance. Another target for ICIs is cytotoxic T-lymphocyteassociated protein 4 (CTLA-4), which plays an important role in the regulation of immune activation and tolerance as it binds to cytotoxic lymphocytes that ligate with B-7 expressed on antigen-presenting cells and inhibits T-cell proliferation. This combination of anti-PD-1 and anti-CTLA-4 ICIs demonstrated significant and maintained antitumor efficacy in melanoma and mRCC patients even after their discontinuation ${ }^{[22,23]}$. A recent systematic review and meta-analysis, involving 1644 patients across eight studies involving RCC, demonstrated that tumor PD-L1 expression significantly correlated with OS (HR = 1.98, 95\%CI: 1.22-3.22, Z $=2.77, P=0.006)$ and DFS (HR $=3.70,95 \% \mathrm{CI}: 2.07-6.62, \mathrm{Z}=4.40, P=0.0001)^{[23]}$, suggesting that PD-L1 and PD-1 may be fruitful targets in the adjuvant setting ${ }^{[24]}$.

One of the several ongoing studies is KEYNOTE-564, a randomized phase 3 trial, evaluating pembrolizumab $v s$. placebo for 17 cycles (or one year) of treatment in the setting of intermediate- to highrisk ccRCC or stage M1 with no evidence of disease (M1 NED), no prior systemic treatment for advanced RCC, and disease free after complete or partial nephrectomy (and metastectomy in M1 NED). Patients with M1 disease with a single metastasis that can be completely resected $\leq 1$ year from nephrectomy were also included, and enrolment has completed with 994 patients. At first interim analysis, the primary endpoint of DFS was met with a HR of 0.68 (95\%CI: 0.53-0.87). The estimated DFS rate at 24 months was $77.3 \%$ with pembrolizumab vs. $68.1 \%$ with placebo. In addition, this DFS benefit was seen across all subgroups. Median OS was not reached for both trial cohorts and the $P$-value did not cross the statistical hypothesis testing boundary. The AE profile of pembrolizumab was in line with expectations, with no new safety signals. Allcause AEs of Grades 3-5 occurred in $32.4 \%$ and $17.7 \%$ of patients receiving pembrolizumab and placebo, respectively, while Grades 3-5 TRAEs rates were $18.9 \%$ and $1.2 \%$ in the same respective groups, with no deaths reported in either trial $\operatorname{arm}^{[25]}$.

IMmotiono10 is an ongoing randomized phase 3 trial evaluating atezolizumab, a PD-L1 inhibitor, vs. placebo, with treatment three times weekly for 16 cycles or one year, in the setting of high-risk ccRCC or sarcomatoid (T2 grade 4, T3a grade $3-4, \mathrm{~T} 3 \mathrm{~b} / \mathrm{c}$ any grade, $\mathrm{T} 4$ any grade, or $\mathrm{TxN}+$ any grade) after nephrectomy or complete resection of limited metachronous/synchronous metastasis, and tumor specimens evaluable for PD-L1. The primary endpoint is independent review-assessed DFS. The study also stratifies disease stage (T2/T3a vs. T3b/c/T4/N+vs. metastasectomy) and PD-L1 status on tumor-infiltrating immune cells (IC; PD-L1 IC expression $<1 \% v s . \geq 1 \%$ ). Secondary endpoints include OS, investigator-assessed DFS, independent review-assessed and investigator-assessed DFS in patients with $\geq 1 \%$ PD-L1 IC, disease-specific survival, distant metastasis-free survival, and 3-year rates of independent review-assessed and investigatorassessed DFS. The planned analysis will occur when at least $65 \%$ of patients in the two arms have died and the study has planned to enroll 664 patients $^{[26]}$. 
Another ongoing ICI-adjuvant trial is the Checkmate-914 phase 3 trial evaluating nivolumab (an anti-PD-1 monoclonal antibody) alone or in combination with ipilimumab (an anti-CTLA4 monoclonal antibody) vs. placebo for 24 weeks in the setting of high-risk predominantly ccRCC with pT2a (grade 3 or 4 ), $\mathrm{pT} 2 \mathrm{~b} / \mathrm{pT} 3 / \mathrm{pT} 4$ (any grade), No, or any $\mathrm{T}$ (any grade) N1 after radical or partial nephrectomy with negative surgical margins $>4$ weeks and $\leq 12$ weeks before randomization and no clinical/radiological evidence of macroscopic residual disease or distant metastases post-nephrectomy and tumor tissue obtained $\leq 3$ months pre-enrollment. The primary endpoint is DFS and some of the key secondary endpoints are OS and DFS for nivolumab with ipilimumab $v s$. nivolumab and safety. The planned enrolment is 1600 patients ${ }^{[27]}$.

A further trial involving ICIs is RAMPART, which is a randomized, multi-arm and multi-stage phase 3 trial evaluating durvalumab (an anti-PD-L1 monoclonal antibody), durvalumab plus tremelimumab (an anti CTLA-4 monoclonal antibody), and active monitoring in the setting of intermediate- to high-risk RCC post-nephrectomy. The primary endpoints are DFS and OS, and the two of the key secondary endpoints are metastasis-free survival and RCC-specific survival time. The planned enrolment is 1750 patients ${ }^{[28]}$.

With a novel trial design, PROSPER is a randomized unblinded phase 3 trial evaluating nivolumab in the setting of perioperative stage T2 or greater or node positive Mo RCC of any histology. It is hypothesized that nephrectomy may remove immune effector cells and cytokines from the tumor site, which may result in a decreased immune response, and hence the trial participants will be randomized to receive two doses of neoadjuvant nivolumab pre-nephrectomy with subsequent (adjuvant) nivolumab for 9 months or standard nephrectomy with subsequent observation. The target enrolment is 766 patients, and the primary endpoint is $\mathrm{RFS}^{[29]}$.

\section{Mammalian target of rapamycin inhibitors}

Another type of agent that is being investigated in the adjuvant RCC setting is the mammalian target of rapamycin inhibitor. The EVEREST randomized phase 3 trial is evaluating everolimus in the post radical or partial nephrectomy setting, and it is estimated to complete enrolment by the end of 2021 . The primary endpoint is RFS, and a key secondary endpoint is $\mathrm{OS}^{[30]}$.

\section{CONCLUSION}

The landscape of adjuvant treatment in RCC is likely to change in the next few years, as ICI trials report results, despite the initial disappointment with TKIs. The advent of ICIs and newer generation TKIs has led to a rapid change in the treatment landscape of mRCC, with ICI combinations or ICI-and-TKI combinations being used more widely after clinical trials have demonstrated their superior efficacy to sunitinib. This has renewed hope that some of the current adjuvant trials will yield positive results and become incorporated into the management of non-mRCC patients.

Nevertheless, we are yet to develop a better prognostic tool beyond pathological staging to help select those who are more likely to benefit from adjuvant treatment. In a study by George et al ${ }^{[32]}, 10 \mathrm{SNPs}$ (single nucleotide polymorphisms) in genes associated with RCC tumorigenesis and 3 SNPs (genotype for VEGFR1, VEGFR-2, and endothelial nitric oxide synthase) were found to be associated with statistically significant DFS improvement favoring sunitinib over placebo. In an analysis of sunitinib and placebo groups combined, they also found a significantly longer OS when there was no CCDC26 rs60315789 insertion as compared to the heterozygous genotype, pointing to its potential as a post-surgical prognostic biomarker ${ }^{[31,32]}$. Similarly, in the ARISER trial, those with higher CA-IX expression were shown to have an improved DFS that approached statistical significance. Furthermore, as has been demonstrated in other solid tumors, those with high PD-L1 expression are more likely to benefit more from ICI treatment $t^{[9]}$. 
There is the potential for adverse effects with any therapy; hence, to avoid unnecessary harm, it is crucial to carefully select patients who are most likely to derive benefit from a given treatment. In fact, we have learned from previous adjuvant trials involving VEGFR TKIs that the TRAEs were significant and impacting on the quality of life of the participants, and, more often than not, they resulted in dose reductions, thus diminishing the ability of patients to remain on treatment. Therefore, it is hoped that future studies will help develop predictive biomarkers which can be used in conjunction with pathological staging and risk prognostication to guide patient selection, allowing adjuvant treatment to only be given to those who will most likely benefit from it.

\section{DECLARATIONS}

\section{Authors' contributions}

Made substantial contributions to literature review required for the manuscript as well as writing and editing it: Sawhney P, Suyanto S

Contributed to writing and editing the manuscript: Michael A, Pandha $\mathrm{H}$

\section{Availability of data and materials}

Not applicable.

\section{Financial support and sponsorship}

None.

\section{Conflicts of interest}

Suyanto S is employed by Eisai Inc., but this review paper does not represent the view of Eisai Inc. Suyanto $\mathrm{S}$ is affiliated with Royal Surrey County Hospital. The remaining authors declared that there are no conflicts of interest

\section{Ethical approval and consent to participate}

Not applicable.

\section{Consent for publication}

Not applicable.

\section{Copyright}

(c) The Author(s) 2021.

\section{REFERENCES}

1. Frank I, Blute ML, Cheville JC, Lohse CM, Weaver AL, Zincke H. An outcome prediction model for patients with clear cell renal cell carcinoma treated with radical nephrectomy based on tumor stage, size, grade and necrosis: the SSIGN score. J Urol 2002;168:2395400. DOI PubMed

2. Parker WP, Cheville JC, Frank I, et al. Application of the stage, size, grade, and necrosis (SSIGN) score for clear cell renal cell carcinoma in contemporary patients. Eur Urol 2017;71:665-73. DOI PubMed PMC

3. Zisman A, Pantuck AJ, Dorey F, et al. Improved prognostication of renal cell carcinoma using an integrated staging system. $J$ Clin Oncol 2001;19:1649-57. DOI PubMed

4. Kattan MW, Reuter V, Motzer RJ, Katz J, Russo P. A postoperative prognostic nomogram for renal cell carcinoma. J Urol 2001;166:63-7. PubMed

5. Leibovich BC, Blute ML, Cheville JC, et al. Prediction of progression after radical nephrectomy for patients with clear cell renal cell carcinoma: a stratification tool for prospective clinical trials. Cancer 2003;97:1663-71. DOI PubMed

6. Pizzocaro G, Piva L, Colavita M, et al. Interferon adjuvant to radical nephrectomy in Robson stages II and III renal cell carcinoma: a multicentric randomized study. J Clin Oncol 2001;19:425-31. DOI PubMed

7. Messing EM, Manola J, Wilding G, et al; Eastern Cooperative Oncology Group/Intergroup trial. Phase III study of interferon alfa-NL as adjuvant treatment for resectable renal cell carcinoma: an Eastern Cooperative Oncology Group/Intergroup trial. J Clin Oncol 2003;21:1214-22. DOI PubMed

8. Passalacqua R, Caminiti C, Buti S, et al; POLAR-01 Trial Investigators. Adjuvant low-dose interleukin-2 (IL-2) plus interferon- $\alpha$ 
(IFN- $\alpha$ ) in operable renal cell carcinoma (RCC): a phase III, randomized, multicentre trial of the Italian Oncology Group for Clinical Research (GOIRC). J Immunother 2014;37:440-7. DOI PubMed

9. Chamie K, Donin NM, Klöpfer P, et al. Adjuvant weekly girentuximab following nephrectomy for high-risk renal cell carcinoma: the ARISER randomized clinical trial. JAMA Oncol 2017;3:913-20. DOI PubMed PMC

10. Choueiri TK, Kaelin WG Jr. Targeting the HIF2-VEGF axis in renal cell carcinoma. Nat Med 2020;26:1519-30. DOI PubMed

11. Rini BI, Small EJ. Biology and clinical development of vascular endothelial growth factor-targeted therapy in renal cell carcinoma. $J$ Clin Oncol 2005;23:1028-43. DOI PubMed

12. Almeida DV, Oliveira CZ, Soares A, et al; Latin America Cooperative Oncology Group - Genitourinary (LACOG-GU). Meta-analysis of randomized clinical trials (RCT) for the adjuvant treatment of renal cell carcinoma (RCC) with vascular endothelial growth factor receptor tyrosine-kinase inhibitors (VEGFR TKIs). J Clin Oncol 2019;37:4579. DOI

13. Motzer RJ, Hutson TE, Tomczak P, et al. Sunitinib versus interferon alfa in metastatic renal-cell carcinoma. N Engl J Med 2007;356:115-24. DOI PubMed

14. Choueiri TK, Hessel C, Halabi S, et al. Cabozantinib versus sunitinib as initial therapy for metastatic renal cell carcinoma of intermediate or poor risk (Alliance A031203 CABOSUN randomised trial): progression-free survival by independent review and overall survival update. Eur J Cancer 2018;94:115-25. DOI PubMed PMC

15. Sternberg CN, Davis ID, Mardiak J, et al. Pazopanib in locally advanced or metastatic renal cell carcinoma: results of a randomized phase III trial. J Clin Oncol 2010;28:1061-8. DOI PubMed

16. Hutson TE, Al-Shukri S, Stus VP, et al. Axitinib versus sorafenib in first-line metastatic renal cell carcinoma: overall survival from a randomized phase III trial. Clin Genitourin Cancer 2017;15:72-6. DOI PubMed

17. Haas NB, Manola J, Uzzo RG, et al. Adjuvant sunitinib or sorafenib for high-risk, non-metastatic renal-cell carcinoma (ECOG-ACRIN E2805): a double-blind, placebo-controlled, randomised, phase 3 trial. Lancet 2016;387:2008-16. DOI PubMed PMC

18. Motzer RJ, Ravaud A, Patard JJ, et al. Adjuvant sunitinib for high-risk renal cell carcinoma after nephrectomy: subgroup analyses and updated overall survival results. Eur Urol 2018;73:62-8. DOI PubMed PMC

19. Motzer RJ, Haas NB, Donskov F, et al; PROTECT investigators. Randomized phase III trial of adjuvant pazopanib versus placebo after nephrectomy in patients with localized or locally advanced renal cell carcinoma. J Clin Oncol 2017;35:3916-23. DOI PubMed PMC

20. Gross-Goupil M, Kwon TG, Eto M, et al. Axitinib versus placebo as an adjuvant treatment of renal cell carcinoma: results from the phase III, randomized ATLAS trial. Ann Oncol 2018;29:2371-8. DOI PubMed PMC

21. Eisen T, Frangou E, Oza B, et al. Adjuvant sorafenib for renal cell carcinoma at intermediate or high risk of relapse: results from the SORCE randomized phase III intergroup trial. J Clin Oncol 2020;38:4064-75. DOI PubMed PMC

22. Gul A, Rini B. Adjuvant therapy in renal cell carcinoma. Cancer 2019;125:2935-44. DOI PubMed

23. Larkin J, Chiarion-Sileni V, Gonzalez R, et al. Five-year survival with combined nivolumab and ipilimumab in advanced melanoma. $N$ Engl J Med 2019;381:1535-46. DOI PubMed

24. Shen M, Chen G, Xie Q, et al. Association between PD-L1 expression and the prognosis and clinicopathologic features of renal cell carcinoma: a systematic review and meta-analysis. Urol Int 2020;104:533-41. DOI PubMed

25. Choueiri TK, Tomczak P, Park SH, et al. Pembrolizumab versus placebo as post-nephrectomy adjuvant therapy for patients with renal cell carcinoma: randomized, double-blind, phase III KEYNOTE-564 study. J Clin Oncol 2021;39:LBA5. DOI

26. Uzzo R, Bex A, Rini BI, et al. A phase III study of atezolizumab (atezo) vs placebo as adjuvant therapy in renal cell carcinoma (RCC) patients (pts) at high risk of recurrence following resection (IMmotion010). J Clin Oncol 2017;35:TPS4598. DOI

27. Bex A, Russo P, Tomita Y, et al. A phase III, randomized, placebo-controlled trial of nivolumab or nivolumab plus ipilimumab in patients with localized renal cell carcinoma at high-risk of relapse after radical or partial nephrectomy (CheckMate 914). J Clin Oncol 2020;38:TPS5099. DOI

28. Renal adjuvant multiple arm randomised trial (RAMPART). Available from: https://clinicaltrials.gov/ct2/show/NCT03288532. [Last accessed on 7 Jul 2021].

29. Haas NB, Puligandla M, Allaf ME, et al. PROSPER: phase III randomized study comparing perioperative nivolumab versus observation in patients with renal cell carcinoma (RCC) undergoing nephrectomy (ECOG-ACRIN EA8143). J Clin Oncol 2020;38:TPS5101. DOI

30. S0931, everolimus in treating patients with kidney cancer who have undergone surgery (S0931). Available from: https://clinicaltrials.gov/ct2/show/NCT01120249 [Last accessed on 7 Jul 2021].

31. Buller DM, Ristau BT. Improving patient selection for adjuvant therapy in high-risk renal cell carcinoma. Ann Transl Med 2019;7:S104. DOI PubMed PMC

32. George DJ, Martini JF, Staehler M, et al. Phase III trial of adjuvant sunitinib in patients with high-risk renal cell carcinoma: exploratory pharmacogenomic analysis. Clin Cancer Res 2019;25:1165-73. DOI PubMed PMC 